( -10 fold, $\mathrm{p}<0.05)$. The combination of IL-17A or F with TNF induced activation of HIF1- $\alpha$ under normoxic conditions (2 fold, $\mathrm{p}=0.003$ ).

Conclusion When combined with TNF, IL-17A and F induced very similar but not identical expression pattern in RA synoviocytes, may contribute to the progression of RA through increased synoviocyte aggressiveness. Part of this effect results from a mediated CXCR4/SDF1 pathway. These results are in line with IL-17A and F targeting in RA.

\section{A70 IL-17A AND IL-17F INDUCE AN INVASIVE PHENOTYPE IN HUMAN RHEUMATOID SYNOVIOCYTES THROUGH THE SAME SIGNALING PATHWAY AND THE CXCR4/SDF1 AXIS}

Arnaud Hot, Pierre Miossec Immunogenomics and Inflammation Research unit and Department of Immunology and Rheumatology, Hospital Edouard Herriot, Lyon, France

\subsection{6/ard.2010.149104.14}

Objective IL-17A is implicated in rheumatoid arthritis (RA) pathogenesis; but the contribution of IL-17F remains to be clarified. This report analyses the effects IL-17A versus IL-17F on gene expression, signaling and invasiveness in human RA synoviocytes.

Methods The comparison between IL-17A and F effects on RA synoviocytes was assessed at the mRNA level by microarrays (Affymetrix U133+2). Western blotting, qRT-PCR, and DNA binding assay were used to evaluate their signaling pathways. The capacities of IL-17A and F alone or in combination with TNF to induce synoviocyte migration and invasion were tested using transwell Matrigel invasion chambers. A functional DNA binding assay was used to evaluate the regulation of Hypoxia Induced Factor 1 (HIF1- $\alpha$ ) activation.

Results In microarrays, IL-17A and IL-17F alone had very similar but not identical regulatory effects, IL-17F being less active, with a synergistic pattern in the presence of TNF. This synergistic effect was linked in part to the enhancing expression of TNF RII by IL-17A and F. Regarding their signaling pathway, virtually all IL17A and F inducible genes were dependent on NF- $\kappa \mathrm{B}$ activation, whereas a minor number was modulated by $\mathrm{p} 38$. Hypoxia-induced pathway was activated by IL-17A and F. Among the hypoxia-induced genes, IL-17A and $\mathrm{F}$ alone or combined with TNF induced CXCR4 mRNA (289 fold for IL17A, 34 fold for IL-17F, 450 fold for the combination of IL-17A with TNF). Over expression of CXCR4 at the surface of synoviocytes was confirmed by IF staining. IL-17A and TNF induced in synergy synoviocyte migration and invasion through CXCR4 (6 vs 67 migrated cells/HPF, $\mathrm{p} \leq 0.05)$. Blockade of CXCR4 decreased synoviocyte migration 